\title{
CLINICAL STUDY OF THE IMPACT ON OVARIAN RESERVE BY DIFFERENT HEMOSTASIS METHODS IN LAPAROSCOPIC CYSTECTOMY FOR OVARIAN ENDOMETRIOMA
}

\author{
By \\ Ismail A. Mira, Ihab H. Mohamed and Ibrahim M. Mohamed \\ Department of Obstetrics and Gynecology, Faculty of Medicine, Al-Azhar University \\ Corresponding author: Ibrahim M. Mohamed, \\ Mobile: 01020501292, E-mail: $\underline{\text { dr_ibrahim_mohamed20@gmail.com }}$
}

\begin{abstract}
Background: Endometriosis is the presence of endometrial glands or stroma in sites other than the uterine cavity such as the ovaries, fallopian tubes and the pelvis. The most common location of endometriosis is the ovary, occurring in $17-44 \%$ of patients affected by endometriosis.
\end{abstract}

Objectives: To explore the impact of laparoscopic ovarian cystectomy versus aspiration and coagulation on ovarian reserve in cases of ovarian endometrioma.

Patients and Methods: This randomized controlled prospective study was conducted on ninety two patients presenting with unilateral ovarian endometrioma at Obstetrics and Gynecology Department, Al-Azhar University Hospitals during the period from April 2018 to October 2019. FSH, LH, E2, AMH, AFC (antral follicular count); all were determined before and after laparoscopic surgery (postoperative three months); for two groups: Group A for cystectomy and Group B for aspiration and coagulation, performed randomly using closed envelope. Histopathological examination was done to the cystectomy group to confirm endometriosis and detect the presence of healthy ovarian tissue in the excised cyst wall.

Results: There were postoperative significant decreases in FSH, LH, E2, AMH and AFC in each group compared with the preoperative values but were within normal ranges, and this decrease was of no statistically significant difference between both groups as regarding (FSH, LH, E2, AMH). There was a statistically significant difference between both groups in AFC which was more decreased in the cystectomy group. So, AFC was more accurate in predicting ovarian reserve loss than AMH.

Conclusion: Ovarian reserve decreased by both techniques; but the decrease was more frequent in cystectomized ovaries.

Keywords: Ovarian endometrioma, laparoscopic surgery, ovarian reserve

\section{INTRODUCTION}

Endometriosis is an enigmatic disease characterized by the development of the endometrial tissue outside of the uterus. The most common location of endometriosis is the ovary, occurring in $17-44 \%$ of patients affected by endometriosis (De Ziegler et al., 2010).
Because endometriosis is most common in reproductive-age women, it is important to predict and protect the patients' ovarian function. Ovarian reserve is defined as the functional potential of the ovary, and it reflects the number and quality of the follicles left in the ovary at any given time. Available 
tests of ovarian reserve include biochemical markers (FSH, estradiol, antimüllerian hormone and inhibin B) and ovarian ultrasound imaging (antral follicle count and ovarian volume) (Chen et al., 2014).

There is no single assessment of ovarian reserve that has $100 \%$ sensitivity and specificity; tests often are combined in an attempt to improve the prediction of poor outcomes. Antimüllerian hormone (AMH) and antral follicle count (AFC) are the most accurate predictors (Practice Committee of the American Society for Reproductive Medicine, 2012).

Care of the endometriosis patient today can be complex and difficult. Once medical management has been exhausted and symptoms persist patients desire further treatment options. Surgical techniques, success rates, patient satisfaction, and risks must be discussed and understood at depth before planning patient surgical intervention (Zanelotti and Decherney, 2017).

The most common surgical techniques used in laparoscopic treatment of ovarian endometrioma are stripping technique and cyst aspiration \& coagulation technique and there is still a debate between both techniques which is better to be used. (Tsolakidis et al., 2010).There is a metaanalysis showed that in comparison to drainage and ablative surgery, excision of endometriomas is better in terms of reduced pain, increased pregnancy, and decreased recurrence and reoperation rates (ACOG, 2014).

Laparoscopic excision of endometriomas is regarded as a first-line treatment, but the reduced numbers of retrieved oocytes for in vitro fertilization and premature ovarian failure after surgery reported in several papers have raised concerns (Ruiz-Flores and GarciaVelasco, 2012).

Ovarian reserve decreased by both techniques but the decrease was statistically significant more frequent in cystectomized ovaries than in coagulated ovaries (Var et al., 2011).

Approximately $80 \%$ of cases with ovarian endometrioma excision, some healthy ovarian tissue inevitably had to be removed which was detected in histopathological assessment (Alborzi et al., 2014).

Ovarian damage was shown to increase when endometrioma diameter exceeded 4 cm (Tanget al., 2013).

The aim of this study was to evaluate the impact on ovarian reserve by different hemostasis methods in laparoscopic cystectomy for ovarian endometrioma.

\section{PATIENTS AND METHODS}

This was a prospective randomized controlled trial which was conducted at Al- Azhar University Hospital during the period from April 2018 to October 2019. Ninety two patients with ovarian endometrioma were recruited for this study. This study was approved by ethical committee of the department.

The study included females with age group 20-40 years diagnosed by ultrasound with unilateral ovarian endometrioma $\geq 3 \mathrm{~cm}$.

Patients with a previous ovarian surgery, endocrinological diseases affecting ovarian reserve e.g. Diabetes mellitus, hypothyroidism, PCO (polycystic ovary syndrome), suspicion of 
ovarian malignancy by ultrasound, other pelvic pathology e.g. uterine fibroid, PID and high basal FSH $>10 \mathrm{mIU} / \mathrm{mL}$ were excluded from the study.

\section{All patients were subjected to the following:}

-Full medical history and examination.

-Laboratory investigations included routine preoperative labs: complete blood count, coagulation profile, fasting and postprandial blood sugar, liver and kidney functions tests. Hormonal profile (preoperative on days 2-4 of the menstrual cycle and repeated 3 months postoperative): FSH, LH, E2, AMH. Blood samples were taken on plain tubes, then allowed to clot and sera were separated and stored at $-20 \circ \mathrm{C}$ until assay, $\mathrm{FSH}, \mathrm{LH}$ and E2 were analyzed on AVIDA Centaur by kits purchased from Siemens Healthcare Diagnostic Inc. 511 Benedict Avenue, Tarrytown, NY105915097 USA, AMH was analyzed by ELISA technique with kits purchased from Beckmen Coulter - Germany. The sensitivity of the assay was $0.08 \mathrm{ng} / \mathrm{ml}$.

Transvaginal ultrasound was in married females and abdominal and or transrectal ultrasound in virgins to detect ovarian endometrioma; site, size, number,and antral follicular count (AFC) in the affected ovary and in the normal one on days $2-5$ of the menstrual cycle.

Informed written consent was obtained from all women included in the study.

Operative laparoscopy: The cases were randomly allocated using closed envelope into two groups according to subsequent management; Group A had endometriotic cyst excision (stripping), Group B had cyst aspiration and coagulation.
Laparoscopic operations were performed using video control under general anesthesia. The operations were performed with two to three 5-mm trocars in the lower abdomen and a 10-mm intraumbilical main trocar, and normal saline solution for irrigation.

In the stripping group of the ovary (Group A): After a cleavage plane between the cyst wall and ovarian cortex was identified, the ovary was pulled slowly and gently in opposite directions by means of two atraumatic grasping forceps. After removing the pseudocapsule from the abdominal cavity, the ovarian bed was left open. The cyst wall was histopathologically examined for confirmation of the diagnosis of endometrioma and for detection presence or absence of ovarian tissue (ovarian follicles) in the excised cyst. In the aspiration and coagulation group of the ovary (Group B): Fenestration was first performed, and then the inner cyst wall was coagulated by the touch technique using bipolar forceps. No sutures were used in either group, and the ovary was left open. Postoperatively: \# the pathology results were examined to confirm endometriosis and detect the presence of healthy ovarian tissue in the excised cyst wall and number of ovarian follicles if present. Hormone profile was repeated after 3months (FSH, LH, E2,AMH), \# Antral follicular count was repeated by ultrasound 3 months postoperative. Pelvic pain was reassessed by Visual Analogue Scale (VAS) of pain scoring system after 3 months of surgery. Primary outcome (Most important outcomes to be assessed). To evaluate the impact of laparoscopic ovarian cystectomy versus aspiration and 
coagulation in cases of ovarian endometrioma on ovarian reserve.

To evaluate the impact of laparoscopic ovarian cystectomy versus aspiration and coagulation in cases of ovarian endometrioma on chronic pelvic pain.

\section{Statistical analysis:}

Data were coded and entered using the statistical package SPSS (Statistical Package for the Social Sciences) version 24. Data were summarized using mean, standard deviation, median, minimum and maximum in quantitative data and using frequency (count) and relative frequency (percentage) for categorical data.
Comparisons between the two groups were done using unpaired $t$ test in normally distributed quantitative variables while non-parametric Mann-Whitney test was used for non-normally distributed quantitative variables. For comparison of serial measurements within each patient (pre and post) paired t test or the nonparametric Wilcoxon signed rank test were used. For comparing categorical data, Chi square ( $\square 2$ ) test was performed. Exact test was used instead when the expected frequency is less than 5. Pvalues less than 0.05 were considered as statistically significant.

\section{RESULTS}

There was NO statistical significant difference between ovarian cystectomy group (group A) and aspiration group (group $\mathrm{B}$ ) regarding to age ( $\mathrm{P}$ value > 0.05). There was no statistically significant difference between cystectomy group (Group A) and aspiration group (Group B) as regard marital status, parity and clinical presentation. ( $\mathrm{P}$ value $>0.05$ ) (Table 1).

Table (1): Comparison between ovarian cystectomy group and aspiration group as regard age, marital status, parity and clinical presentation

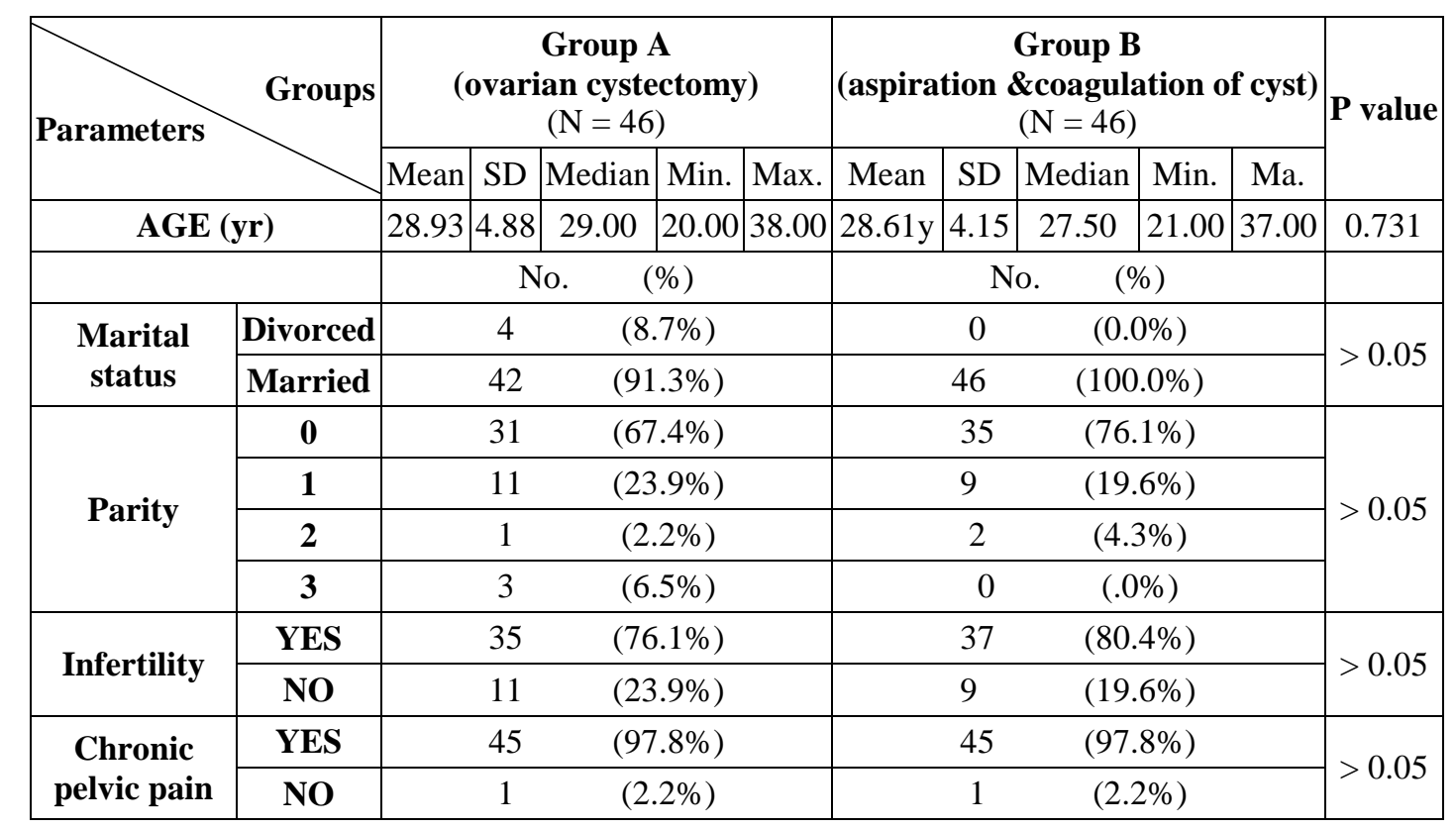


There was a statistically significant difference in the cystectomy group (Group A) as regards preoperative \& postoperative (FSH, LH, E2, AMH, AFC in the affected ovaries and total AFC and) as all these parameters decreased postoperative but within normal ranges (FSH, LH, E2, AMH, AFC). (P value < $0.05)$. This means that ovarian reserve was decreased. There was a statistically significant difference in the aspiration group (Group B) as regards preoperative \&postoperative (FSH, LH, E2, AMH, AFC in the affected ovaries and total AFC) as all these parameters decreased postoperative but within normal ranges $(\mathrm{P}$ value $<0.05)$. This means that ovarian reserve was decreased (Table 2).

Table (2): Comparison between pre and post operative (hormone profile, AFC) in the ovarian cystectomy group (Group A) and in the aspiration \&coagulation group (Group B)

\begin{tabular}{|c|c|c|c|c|c|}
\hline \multirow{2}{*}{ Parameters } & \multicolumn{2}{|c|}{ Preoperative } & \multicolumn{2}{c|}{ Postoperative } & \multirow{2}{*}{ P value } \\
\cline { 2 - 5 } FSH (mIU/ml) & Mean & \pm SD & Mean & \pm SD & \\
\hline LH (mIU/ml) & 7.12 & \pm 1.30 & 6.25 & \pm 1.49 & $<0.001$ \\
\hline E2 (pg/ml) & 4.89 & \pm 1.06 & 4.10 & \pm 1.08 & $<0.001$ \\
\hline AMH (ng/ml) & 3.24 & \pm 1.85 & 1.76 & \pm 1.18 & $<0.001$ \\
\hline AFC affected ovary & 7.65 & \pm 1.10 & 4.48 & \pm 0.94 & $<0.001$ \\
\hline AFC normal ovary & 8.04 & \pm 1.52 & 7.96 & \pm 1.41 & $>0.05$ \\
\hline Total AFC & 15.70 & \pm 2.24 & 12.46 & \pm 2.03 & $<0.001$ \\
\hline FSH (mIU/ml) & 6.67 & \pm 2.22 & 6.36 & \pm 2.11 & $<0.001$ \\
\hline LH (mIU/ml) & 4.57 & \pm 1.61 & 4.37 & \pm 1.60 & $<0.001$ \\
\hline E2 (pg/ml) & 43.10 & \pm 12.77 & 41.46 & \pm 12.56 & $<0.001$ \\
\hline AMH (ng/ml) & 3.07 & \pm 2.25 & 2.52 & \pm 2.04 & $<0.001$ \\
\hline AFC affected ovary & 7.39 & \pm 1.16 & 5.67 & \pm 1.17 & $<0.001$ \\
\hline AFC normal ovary & 8.22 & \pm 1.52 & 8.13 & \pm 1.53 & $>0.05$ \\
\hline Total AFC & 15.63 & \pm 2.30 & 13.80 & \pm 2.24 & $<0.001$ \\
\hline
\end{tabular}

There was a postoperative decrease in all hormone profile parameters (FSH,LH,E2,AMH) in both groups compared with the preoperative values, but this decrease was of NO statistically significant difference between Group A and Group B before and after operation $(\mathrm{P}$ value $>0.05)$. There was NO statistically significant difference between Group A and Group B as regard preoperative ultrasound chocolate cyst mean diameter $(\mathrm{P}$ value $>0.05)$ (Table 3). 
Table (3): Comparison between ovarian cystectomy group and aspiration group as regards hormone profile (FSH, LH, E2, AMH) before and after operation, preoperative ultrasound chocolate cyst mean diameter

\begin{tabular}{|c|c|c|c|c|c|}
\hline \multirow{2}{*}{ Parameters } & \multicolumn{2}{c|}{$\begin{array}{c}\text { Group A } \\
\text { Groups }\end{array}$} & \multicolumn{2}{c|}{$\begin{array}{c}\text { Group B } \\
\text { (aspiration \& } \\
\text { coagulation of cyst) }\end{array}$} & \multirow{2}{*}{ P value } \\
\cline { 2 - 5 } & Mean & \pm SD & Mean & \pm SD & \\
\hline Pre:FSH (mIU/ml) & 7.12 & \pm 1.30 & 6.67 & \pm 2.22 & $>0.05$ \\
\hline Post:FSH (mIU/ml) & 6.25 & \pm 1.49 & 6.36 & \pm 2.11 & $>0.05$ \\
\hline Pre:LH (mIU/ml) & 4.89 & \pm 1.06 & 4.57 & \pm 1.61 & $>0.05$ \\
\hline Post:LH (mIU/ml) & 4.10 & \pm 1.08 & 4.37 & \pm 1.60 & $>0.05$ \\
\hline Pre:E2 (pg/ml) & 44.76 & \pm 14.19 & 43.10 & \pm 12.77 & $>0.05$ \\
\hline Post:E2 (pg/ml) & 42.99 & \pm 14.04 & 41.46 & \pm 12.56 & $>0.05$ \\
\hline Pre:AMH (ng/ml) & 3.24 & \pm 1.85 & 3.07 & \pm 2.25 & $>0.05$ \\
\hline Post:AMH (ng/ml) & 1.76 & \pm 1.18 & 2.52 & \pm 2.04 & $>0.05$ \\
\hline $\begin{array}{c}\text { Preopertative US } \\
\text { ( chocolate cyst mean } \\
\text { diameter) (cm) }\end{array}$ & 5.42 & \pm 1.20 & 5.13 & \pm 0.97 & $>0.05$ \\
\hline
\end{tabular}

There was a statistically significant difference between both groups as regard postoperative (AFC in the affected ovaries and total AFC) as both decreased in both groups but the decrease was more in Group A (the cystectomy group) (P value $<0.05)$. There was no statistically significant difference between between Group A and Group B as regards preoperative and postoperative (AFC of normal ovary), also there was NO statistically significant difference between both groups as regard preoperative (total AFC) $(\mathrm{P}$ value $>0.05)$ (Table 4).

Table (4): Comparison between ovarian cystectomy group and aspiration group as regards ultrasound AFC (antral follicular count) pre and post operative

\begin{tabular}{|c|c|c|c|c|c|}
\hline \multirow{2}{*}{ Parameters } & \multicolumn{2}{|c|}{$\begin{array}{c}\text { Group A } \\
\text { (ovarian cystectomy) }\end{array}$} & \multicolumn{2}{c|}{$\begin{array}{c}\text { Group B } \\
\text { (aspiration \& } \\
\text { coagulation of cyst) }\end{array}$} & \\
\cline { 2 - 6 } & Mean & \pm SD & Mean & \pm SD & P value \\
\hline $\begin{array}{c}\text { pre: AFC affected } \\
\text { ovary }\end{array}$ & 7.65 & \pm 1.10 & 7.39 & \pm 1.16 & $>0.05$ \\
\hline $\begin{array}{c}\text { pre: AFC normal } \\
\text { ovary }\end{array}$ & 8.04 & \pm 1.52 & 8.22 & \pm 1.52 & $>0.05$ \\
\hline pre:Total AFC & 15.70 & \pm 2.24 & 15.63 & \pm 2.30 & $>0.05$ \\
\hline $\begin{array}{c}\text { post: AFC affected } \\
\text { ovary }\end{array}$ & 4.48 & \pm 0.94 & 5.67 & \pm 1.17 & $<\mathbf{0 . 0 0 1}$ \\
\hline $\begin{array}{c}\text { post :AFC normal } \\
\text { ovary }\end{array}$ & 7.96 & \pm 1.41 & 8.13 & \pm 1.53 & $>0.05$ \\
\hline post: Total AFC & 12.46 & \pm 2.03 & 13.80 & \pm 2.24 & $>0.05$ \\
\hline
\end{tabular}


There was a little help given by histopathological assessment to detect presence of any ovarian follicles in the cyst wall of the cystectomy group as we found ovarian follicles in the cyst wall in 5 cases with 2 follicles (the follicles were primary follicles in 2 cases and primoridal follicles in the rest 3 cases) also we found 2 cases with 3 primoridal follicles, while the rest of cases (39) showed no follicle loss at all. Only 7 cases out of 46 cases $(15.2 \%)$ detected loss of follicles by histopathological examination, which was not matching with the marked decrease of postoperative AFC in the affected ovary of this group compared with preoperative values (Table 5).

Table (5): Number of ovarian follicles detected by pathological assessment of the cyst wall of cystectomy group (Group A)

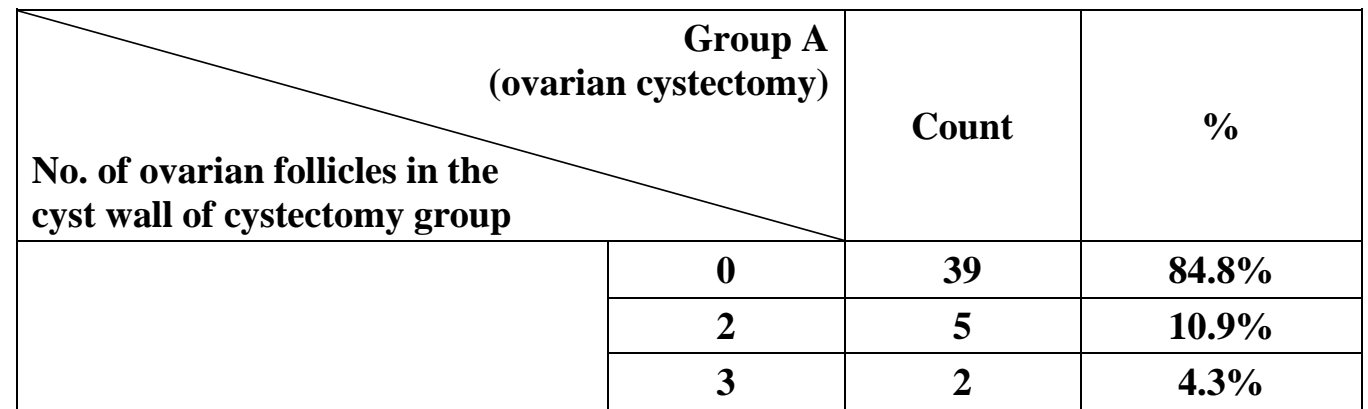

\section{DISCUSSION}

Our study partially agrees with a study done by Var et al. (2011) who, concluded that ovarian reserve (detected by FSH, AMH \& AFC) decreased by both technique, but the decrease was statistically significantly more frequent in cystectomized ovaries than in coagulated ovaries. This agreed with our study in the significant difference in AFC between both groups.

The disagreement was about the insignificant difference in FSH and $\mathrm{AMH}$ between both groups in our study. The explanation for that may be due to different inclusion criteria as they choose women with chocolate cyst measuring five centimeters or more whether unilateral or bilateral.

Our observation agreed with a metaanalysis done done by Raffi et al. (2012) that suggested a negative impact of excision of endometriomas on ovarian reserve as evidenced by a significant postoperative fall in circulating $\mathrm{AMH}$. This may be due to similar mean age group and sample size.This meta-analysis included 8 studies (Ercan et al. 2010, Biacchiardi et al. 2011, Ercan et al. 2011, Hirokawa et al. 2011, Hwu et al. 2011, Kitajima et al. 2011, Lee et al. 2011, and Raffi et al., 2012).

Biacchiardi et al. (2011) agreed that serum AMH concentrations significantly decreased after the cystectomy operation there was no significant differences found in basal FSH, LH and estradiol concentrations before and after surgery. Also, they found that the AFC in the affected ovary appeared to be higher 3 months after laparoscopy than before the operation, although not to a statistically significant level. This discrepancy in results may be due to their higher mean of age which was 34.2 years \pm 5.4 and the 
presence of unilateral and bilateral endometriomas in their inclusion criteria.

Agreeing with our results Var et al. (2011) compared the AFC in two groups, coagulated and cystectomy groups for bilateral ovarian endometrioma. The study showed, that in the coagulated ovary, the precoagulation number had fallen by the postcoagulation stage. The difference was statistically significant. In the cystectomized ovaries, the preoperative number of follicles fell in the postcystectomy stage. The difference was statistically significant. The antral follicle numbers fell after both operations.

On the other hand, the study by Alborzi et al. (2014) disagreed with our study; As they found a significant decrease of serum AMH 1st week post-operative then a significant increase in serum AMH level 3 months after surgery, which could be explained by a good healing process and reperfusion of the operated ovaries. Also they found a significant increase in serum FSH level in all patients which was more remarkable in patients older than 38 years. Although the AFC increased significantly, 3 months after the operation, but the level of E2 remained unchanged.

On the contrary to our study Van Disseldorp et al. (2010), studied inter- and intra-cycle variability of anti-Mullerian hormone and antral follicle counts and found no any significant reduction in AFC, which is another marker of ovarian reserve that correlates well with circulating AMH. This may be attributed to a beta-error due to the small numbers included in this analysis. Furthermore, AFC can be difficult to assess in the presence of endometriomas and has also been shown to be less reproducible than AMH.

In the present study, as regarding the histopathological assessment for detection of any ovarian follicles in the cyst wall of the cystectomy group, we found ovarian follicles in the cyst wall in 5 cases with 2 follicles (the follicles were primary follicles in 2 cases and primoridal follicles in the rest 3 cases), also we found 2 cases with 3 primoridal follicles, while the rest of cases (39) showed no follicle loss at all. So, only $15.2 \%$ detected loss of follicles by histopathological examination, which was not matching with the marked decrease of postoperative AFC in the affected ovary of this group compared with preoperative values.

Similar results were found by Bhat et al (2014) who showed that loss of follicles as depicted by histopatholgical examination was seen in $27 \%$ of operated cyst wall.

Our study disagreed with a study performed by Hansen et al (2011) who stated that the ovarian AFC and serum levels of AMH correlate with the ovarian primordial follicle number even after adjustment for chronological age. This discrepancy in results may be due to that they performed oophorectomy not ovarian cystectomy, so the injury and loss of follicles in the cyst wall was not present and the detection of follicles was easier.

\section{CONCLUSION}

Ovarian reserve decreased by both techniques, but the decrease was statistically significantly more frequent in cystectomized ovaries than in coagulated ovaries. Antral follicular count (as one of ovarian reserve tests) was more accurate 
in measuring the actual ovarian reserve loss in both groups as there was statistically significant difference between both groups, in comparison with $\mathrm{AMH}$ values which showed no statistically significant difference between both groups.

\section{REFERENCES}

1. Alborzi, S., Keramati, P., Younesi, M., Samsami, A., and Dadras, N. (2014): The impact of laparoscopic cystectomy on ovarian reserve in patients with unilateral and bilateral endometriomas. Fertility and sterility, 101(2): 427-434.

2. American College of Obstetricians and Gynecologists (2014): Female age-related fertility decline. ACOG, Committee Opinion No. 589, Obstet Gynecol 123:719-21.

3. Bhat RG, Dhulked S, Ramachandran A, Bhaktha R, Vasudeva A, Kumar $P$ and Rao AC. (2014): Laparoscopic cystectomy of endometrioma: Good surgical technique does not adversely affect ovarian reserve. Journal of human reproductive sciences.; $7(2): 125$.

4. Bhat, R. G., Dhulked, S., Ramachandran, A., Bhaktha, R., Vasudeva, A., Kumar, P. and Rao, A. C. (2014): Laparoscopic cystectomy of endometrioma: Good surgical technique does not adversely affect ovarian reserve. Journal of Human Reproductive Sciences, 7(2): 125132.

5. Biacchiardi, C. P., Delle Piane, L., Camanni, M., Deltetto, F., Delpiano, E. M., Marchino, G. L., and Revelli, A. (2011): Laparoscopic stripping of endometriomas negatively affects ovarian follicular reserve even if performed by experienced surgeons. Reproductive Biomedicine online, 23(6): 740-746.

6. Chen Y, Pei H, Chang Y, Chen M, Wang H, Xie $\mathbf{H}$ and Yao S. (2014): The impact of endometrioma and laparoscopic cystectomy on ovarian reserve and the exploration of related factors assessed by serum antiMullerian hormone: a prospective cohort study. Journal of ovarian research, 1;7(1):108.

7. De Ziegler D, Borghese B and Chapron C. (2010): Endometriosis and infertility: pathophysiology and management. Lancet, 376(9742):730-738.

8. Ercan CM, Sakinci M, Duru NK and Raffi F. (2010): Antimullerian hormone levels after laparoscopic endometrioma stripping surgery. Gynecol Endocrinol., 26:468-472.

9. Hansen, K. R., Hodnett, G. M., Knowlton, N., and Craig, L. B. (2011): Correlation of ovarian reserve tests with histologically determined primordial follicle number. Fertility and sterility, 95(1): 170-175.

10. Hirokawa, W., Iwase, A., Goto, M., Takikawa, S., Nagatomo, Y., Nakahara, T., and Kikkawa, F. (2011): The post-operative decline in serum anti-Müllerian hormone correlates with the bilaterality and severity of endometriosis. Human Reproduction, 26(4): 904-910.

11. Hwu, Y. M., Wu, F. S. Y., Li, S. H., Sun, F. J., Lin, M. H., and Lee, R. K. K. (2011): The impact of endometrioma and laparoscopic cystectomy on serum antiMüllerian hormone levels. Reproductive Biology and Endocrinology, 9(1): 80-86.

12. Kitajima, M., Khan, K. N., Hiraki, K., Inoue, T., Fujishita, A., and Masuzaki, H. (2011): Changes in serum anti-Müllerian hormone levels may predict damage to residual normal ovarian tissue after laparoscopic surgery for women with ovarian endometrioma. Fertility and Sterility, 95(8): 2589-2591.

13. Lee, D. Y., Young Kim, N., Jae Kim, M., Yoon, B. K., and Choi, D. (2011): Effects of laparoscopic surgery on serum anti-Müllerian hormone levels in reproductive-aged women with endometrioma. Gynecological Endocrinology, 27(10): 733-736.

14. Practice Committee of the American Society for Reproductive Medicine (2012): Testing and interpreting measures of ovarian reserve: a committee opinion. Fertil Steril; 98: 1407-15. 
15. Raffi F, Metwally $M$ and Amer S. (2012): The impact of excision of ovarian endometrioma on ovarian reserve: a systematic review and meta-analysis. J Clin Endocrinol Metab, 97: 3146-54.

16. Ruiz-Flores FJ and Garcia-Velasco JA. (2012): Is there a benefit for surgery in endometrioma-associated infertility?. Current Opinion in Obstetrics and Gynecology, 24(3):136-40.

17. Tang Y, Chen SL, Chen X, He YX, Ye DS, Guo W, Zheng HY and Yang XH. (2013): Ovarian damage after laparoscopic endometrioma excision might be related to the size of cyst. Fertil Steril. 100: 464-469.

18. Tsolakidis D, Pados $G$ and Vavilis $D$. (2010): The impact on ovarian reserve after laparoscopic ovarian cystectomy versus three-stage management in patients with endometriomas: a prospective randomized study: Fertility and Sterility, 94(1): 71-77.
19. Van Disseldorp, J., Lambalk, C. B., Kwee, J., Looman, C. W. N., Eijkemans, M. J. C., Fauser, B. C., and Broekmans, F. J. (2010): Comparison of inter-and intra-cycle variability of anti-Müllerian hormone and antral follicle counts. Human Reproduction, 25(1): 221-227.

20. Var T, Batioglu S, Tonguc E, Kahyaoglu I. (2011): The effect of laparoscopic ovarian cystectomy versus coagulation in bilateral endometriomas on ovarian reserve as determined by antral follicle count and ovarian volume: a prospective randomized study. Fertility and sterility. Jun 1; 95(7):2247-50.

21. Zanelotti A and Decherney A. (2017): Surgery and Endometriosis, Clinical Obstetrics and Gynecology, 60(3): 477-484. 


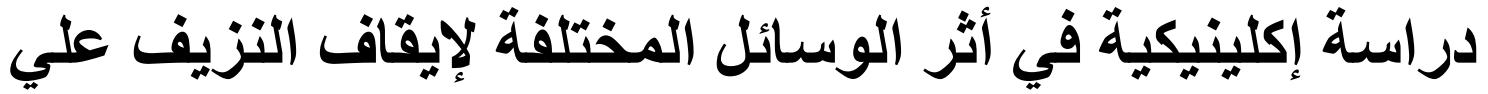

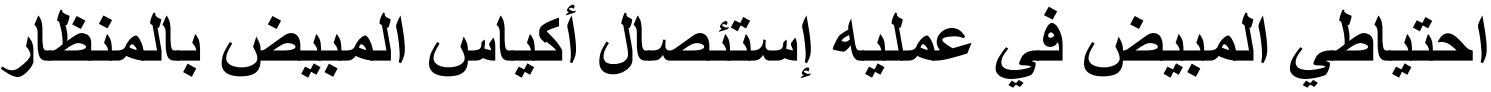

إسماعيل عبد العظيم ميره، إيهاب حساتين محمد، إبراهيم محمد إبراهيم محمد قسم التوليل وأمراض النساء، كلية الطب، جامعة الآزهر

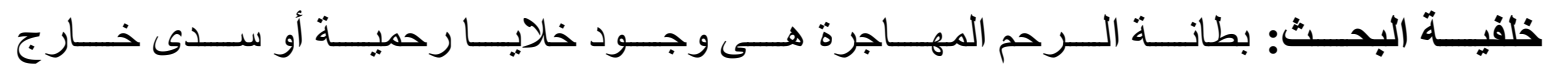

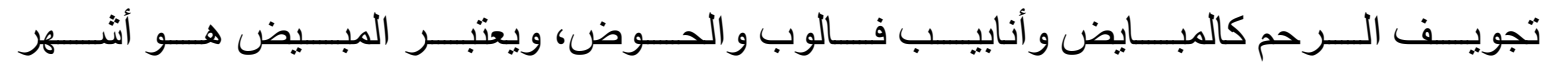

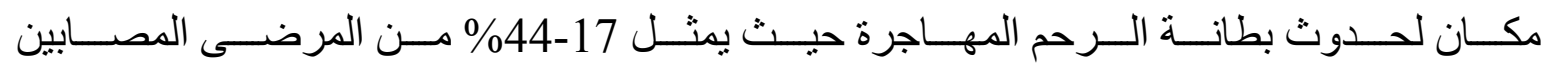
بهذا المرض.

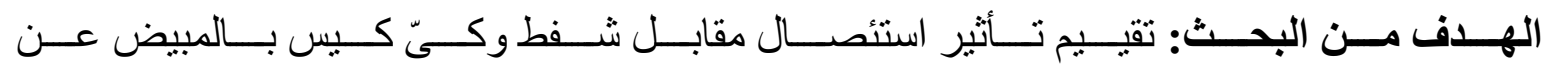
طريق منظار البطن على حتباطي المبيض.

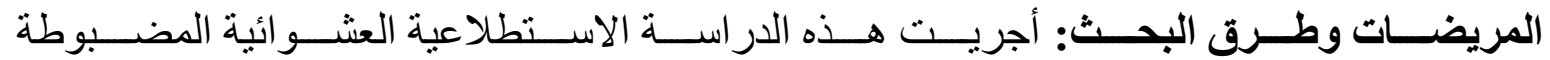

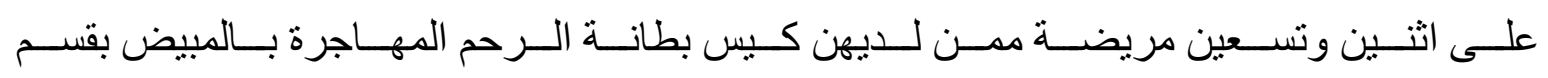

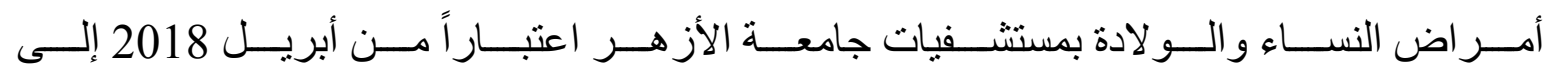

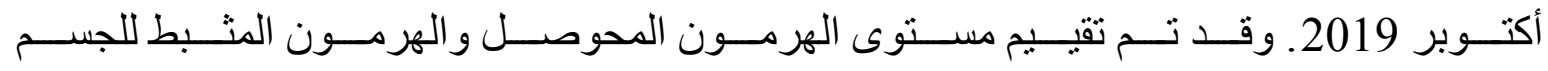

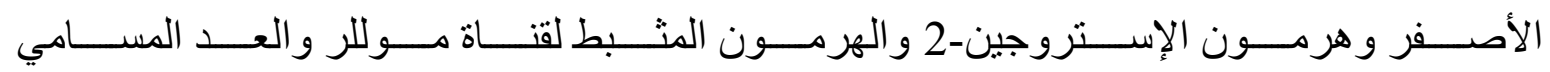

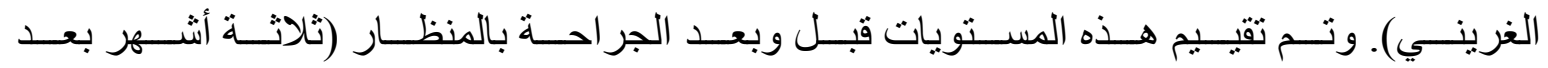

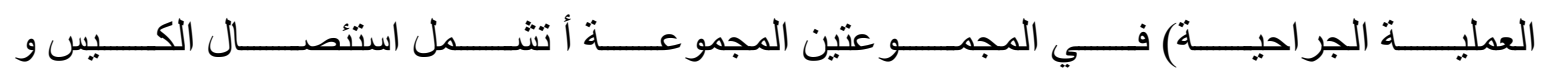

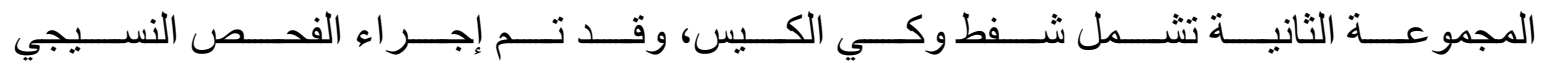

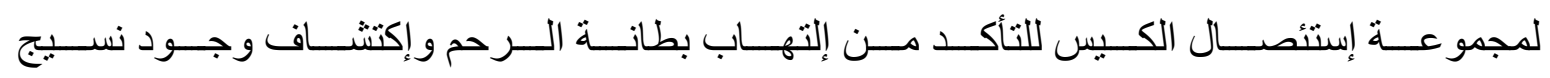
مبيض صحي في جدار المثانة المثير.

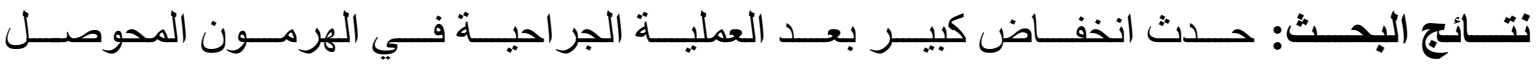

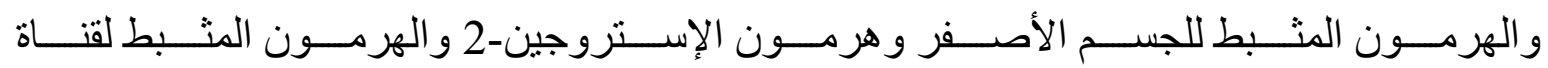

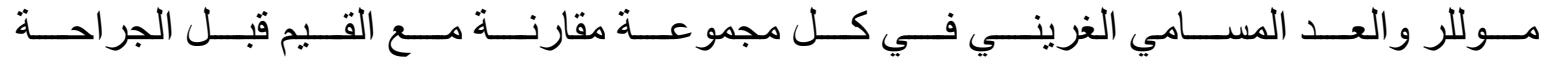

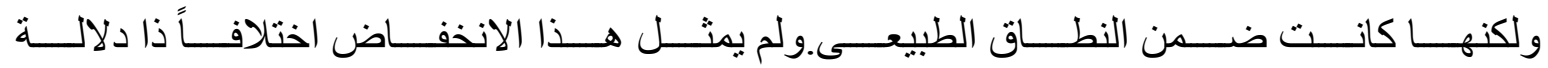

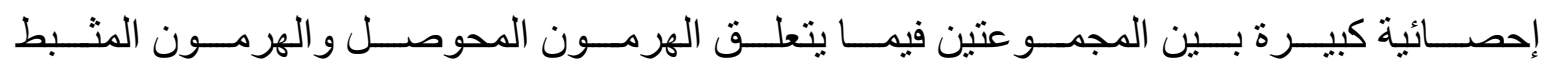

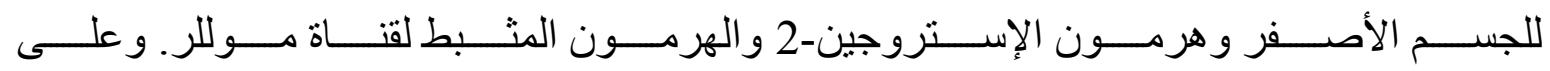




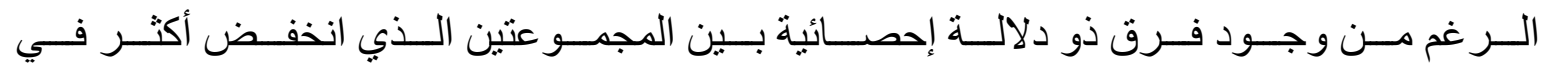

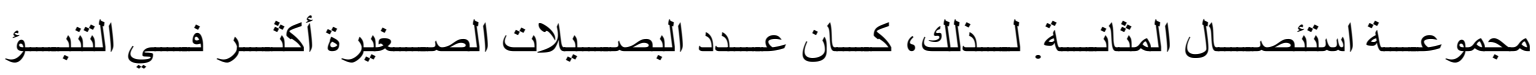
بخسارة احتياطي المبيض من الهرمون المثبط لقناة موللر.

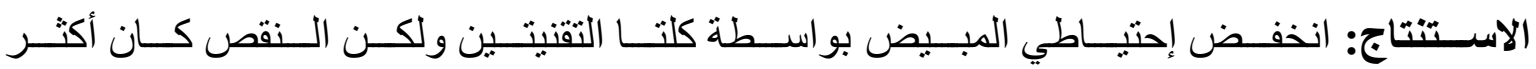
تكراراً في المبايض التي تم استئصسال الكيس منها. 